\title{
Optimal Port Placement in Robot-Assisted Coronary Artery Bypass Grafting
}

\author{
Shaun Selha, B.S. ${ }^{1}$, Pierre Dupont, Ph.D. ${ }^{1}$, Robert Howe, Ph.D. ${ }^{2}$, and \\ David Torchiana, M.D. ${ }^{3}$ \\ 1 Aerospace and Mechanical Engineering, Boston University, Boston MA, USA \\ \{busds, pierre\}@bu.edu \\ 2 Division of Engineering \& Applied Science, Harvard University, Cambridge MA, \\ USA \\ howe@deas . harvard.edu \\ 3 Division of Cardiac Surgery, Massachusetts General Hospital, Boston MA, USA \\ dtorchiana@partners.org
}

\begin{abstract}
A computer-based algorithm is being developed which, using preoperative images, provides the surgeon with a list of feasible port triplets ranked according to tool dexterity and endoscope view quality at each surgical site involved in a procedure. Computer simulation will allow the surgeon to select from among the proposed port locations. The procedure selected for the development of the system consists of left internal mammary artery (LIMA) take-down and bypass grafting. Human trials will begin within a month. Data collected from these trials will be used to validate the system.
\end{abstract}

\section{Introduction}

Robotic assistance enables the use of minimally-invasive techniques in coronary artery bypass grafting by scaling hand motions, decreasing tremor, and enhancing manipulation. In these procedures, endoscopes and instruments are inserted through small incisions or "ports" to access the surgical site. In initial trials of this approach, inappropriate port locations have posed a number of difficulties, including poor dexterity, inability to reach all the required tissue structures, and collisions between the instruments, both within and outside the patient's body.

A graphical simulation system [1] and an algorithmic approach to port placement [2] have been presented in the literature for the daVinci ${ }^{\mathrm{TM}}$ robot system. Experimental guidelines for the selection of port locations for the ZEUS $^{\mathrm{TM}}$ robot system made by Computer Motion, Inc. appear in 3]. This abstract presents an algorithm for optimizing port placement for the ZEUS ${ }^{\mathrm{TM}}$ system.

The three main issues in defining optimal port placement are tool dexterity, endoscope viewpoint and workspace limits. Tool dexterity is defined by the relative orientation of the tools with respect to each other and to the surgical site. Endoscope viewpoint is defined with respect to the tools and with respect to the surgical site. Workspace limits are reached when a desired tool motion would cause a tool / endoscope / patient collision or a robot / robot / patient collision. 


\section{Approach}

At this stage, our efforts have focused on tool dexterity and endoscope viewpoint. Aspects of workspace limits are incorporated through selection of feasible port locations as defined below. The algorithm takes as inputs a CT image of the patient's thorax (with artery locations visualized by contrast agent), an array of possible port locations in the intercostal spaces, and a set of surgical sites where tissue is to be manipulated. Its output is a set of feasible port locations ranked according to a cost function. The latter is based on the optimal orientation of the tools and endoscope with respect to each other and with respect to the surgical sites.

Each surgical site is defined by a point in space and surface normal direction at the point. For LIMA takedown, the spatial curve describing artery location is discretized and the resulting set of points is used for site definition. Feasible port triplets correspond to a subset of array triplets that fall within workspace limits, meet minimum dexterity and viewing requirements and satisfy surgeon preferences. Optimal kinematic dexterity and endoscope viewpoint are defined with respect to a surgical site based on clinical experience. These can be modified according to the procedure, tools and surgeon preferences.

A nested search is used to consider feasible port locations and all surgical sites. Due to the constrained relative positioning of the endoscope and tools, the set of feasible triplets is modest. The result is a ranking of feasible port triplets according to a weighted average of their dexterity measure at all surgical sites. The results are displayed graphically, superimposed on the preoperative image, showing the grid of feasible port locations and surgical sites. The user can select port triplets from among those with highest ranking and manipulate a display of the tools and endoscope. Tool color changes to indicate when dexterity or workspace limits are exceeded. Additional two-dimensional views of the feasible port location grid depict through color-coding their relative optimality to assist the user in comparing port locations.

Data collection during upcoming human trials will permit evaluation of the system. Future work will consider robot interference, robot placement and arm posture as well as the placement of additional assist ports.

\section{References}

1. L. Adhami, E. Coste-Maniere and J.-D. Boissonnat. Planning and simulation of robitically assisted minimal invasive surgery. Proc MICCAI '00. Lecture notes in computer science. Vol. 1935, Springer, October 2000.

2. E. Coste-Maniere, L. Adhami, R. Severac-Bastide, A. Lobontiu, J. Salsibury Jr., J.D. Boisonnat, N. Swarup, G. Guthart, E. Mousseaux and A. Carpentier. Optimized Port Placement for the totally endoscopic coronary artery bypass grafting using the daVinci robotic system. Proc Intl Symp Exp Rob. Springer, December 2000.

3. H. Tabaie, J. Reinbolt, W. Graper, T. Kelly and M. Connor. Endoscopic coronary artery bypass graft (ECABG) procedure with robotic assistance. Heart Surg Forum \#1999-0552, 2:310-317, 1999. 